\title{
SPURIOUS REGRESSIONS WITH TIME-SERIES DATA: FURTHER ASYMPTOTIC RESULTS
}

\author{
David E. A. Giles \\ Department of Economics, University of Victoria \\ Victoria, B.C., Canada V8W 2 Y2
}

August, 2006

(This paper supercedes EWP0203, and is to appear in Communications in Statistics, A.)

\begin{abstract}
A "spurious regression" is one in which the time-series variables are non-stationary and independent. It is well-known that in this context the OLS parameter estimates and the $R^{2}$ converge to functionals of Brownian motions; the "t-ratios” diverge in distribution; and the Durbin-Watson statistic converges in probability to zero. We derive corresponding results for some common tests for the Normality and homoskedasticity of the errors in a spurious regression.
\end{abstract}

Keywords: Spurious regression; normality; homoskedasticity; asymptotic theory; unit roots JEL Classifications: C12, C22, C52

\section{Author Contact:}

David Giles, Dept. of Economics, University of Victoria, P.O. Box 1700, STN CSC, Victoria, B.C., Canada V8W 2Y2; e-mail: dgiles@uvic.ca; FAX: (250) 721-6214 


\section{INTRODUCTION}

Testing and allowing for non-stationary time-series data has been one of the major themes in econometrics over the past quarter-century or so. In their influential and relatively early contribution, Granger and Newbold (1974) drew our attention to some of the likely consequences of estimating a "spurious regression" model. They argued that the "levels" of many economic timeseries are integrated or nearly so, and that if such data are used in a regression model a high $R^{2}$ value is likely to be found even when the series are independent of each other. They also illustrated that the regression residuals are likely to be autocorrelated, as evidenced by a very low value for the Durbin-Watson (DW) statistic. Students of econometrics soon, rather simplistically, equated a "spurious regression" with one in which $R^{2}>D W$. Granger and Newbold (1977) and Plosser and Schwert (1978) added to our awareness and understanding of spurious regressions, but it was Phillips (1986) who provided a formal analytical explanation for the behaviour of the Ordinary Least Squares (OLS) coefficient estimator, the associated $t$-statistics and F-statistic, and the $R^{2}$ and $D W$ statistics in such models.

Phillips (1986) developed a sophisticated asymptotic theory that he used to prove that in a spurious regression, inter alia, the DW statistic converges in probability to zero, the OLS parameter estimators and $R^{2}$ converge weakly to non-standard limiting distributions, and the $t$-ratios and $F$ statistic diverge in distribution as $T \uparrow \infty$. Phillips "solved" the spurious regression problem, and proved that the unfortunate consequences of modelling with integrated data cannot be eliminated by increasing the sample size. This paper uses Phillips' asymptotic theory to demonstrate that the pitfalls of estimating a spurious regression extend to the application of standard diagnostic tests for the normality or homoskedasticity of the model's error term. We prove that the associated test statistics diverge in distribution as the sample size grows, so that one is led inevitably to the false conclusion that there is a "problem" with the usual assumptions about the error term. In fact, the real "problem" is a failure to take account of the non-stationarity of the data when specifying the model. The positive aspect of these results is that they provide us with an extended basis for detecting that we are unwittingly trying to estimate a spurious regression model. 
The next section establishes some of the basic asymptotic results that we use in the later analysis. Section 3 establishes and illustrates the asymptotic behaviour of a well-known omnibus test for normality proposed by Bowman and Shenton (1975), and justified as a Lagrange multiplier test by Jarque and Bera (1987). Two simple variants of the homoskedasticity tests proposed by Breusch and Pagan (1980) and Godfrey (1988) are examined in a similar way in section 4; and some concluding remarks are given in section 5 .

\section{SOME BASIC ASYMPTOTIC RESULTS}

For our purposes, it is sufficient to consider the simple univariate regression model, estimated by Ordinary Least Squares (OLS):

$$
y_{t}=\alpha+\beta x_{t}+u_{t}
$$

The regression is "spurious" because both the dependent variable and the regressor follow independent I(1) processes:

$$
\begin{aligned}
& y_{t}=y_{t-1}+v_{t} \quad ; \quad v_{t} \sim \operatorname{iid}\left(0, \sigma_{v}^{2}\right) \\
& x_{t}=x_{t-1}+w_{t} \quad ; \quad w_{t} \sim \operatorname{iid}\left(0, \sigma_{w}^{2}\right)
\end{aligned}
$$

with $v_{t}$ and $w_{t}$ independent for all t, and (without loss of generality) $v_{0}=w_{0}=0$. In fact [Phillips (1986, p.313)] $v_{t}$ and $w_{t}$ may have heteroskedastic variances, a point that is relevant in section 4 below. So, the true parameter values are $\alpha=\beta=0$.

From Phillips (1986, pp.315 and 326) we know that, by the strong law of McLeish (1975, Theorem 2.10) for weakly dependent sequences, and the Functional Central Limit Theorem [e.g., Hamilton (1994, pp. 479-480)]:

$$
T^{-3 / 2} \sum_{t} x_{t} \Rightarrow \sigma_{w} \int_{0}^{1} W(r) d r=\sigma_{w} \xi_{1}, \quad \text { say }
$$

and

$$
T^{-3 / 2} \sum_{t} y_{t} \Rightarrow \sigma_{v} \int_{0}^{1} V(r) d r=\sigma_{v} \eta_{1}, \quad \text { say }
$$

where $\Rightarrow$ denotes weak convergence of the associated probability measures as $T \uparrow \infty$, and $W(r)$ and 
$V(r)$ are independent Wiener processes on $C[0,1]$, the space of all real-valued functions on $[0,1]$. Using the same approach as Phillips it is also readily shown that

$$
T^{-(k+2) / 2} \sum_{t} x_{t}^{k} \Rightarrow \sigma_{w}^{k} \int_{0}^{1}(W(r))^{k} d r=\sigma_{w}^{k} \xi_{k}, \quad \text { say; } \quad k=1,2,3,4, \ldots \ldots .
$$

and

$$
T^{-(k+2) / 2} \sum_{t} y_{t}^{k} \Rightarrow \sigma_{v}^{k} \int_{0}^{1}(V(r))^{k} d r=\sigma_{v}^{k} \eta_{k}, \quad \text { say } ; \quad k=1,2,3,4, \ldots \ldots . .
$$

From Phillips (1986, p.315) we also know that

$$
\begin{aligned}
& T^{-2} \sum_{t}\left(x_{t}-\bar{x}\right)^{2} \Rightarrow \sigma_{w}^{2}\left(\xi_{2}-\xi_{1}\right)^{2} \\
& T^{-2} \sum_{t}\left(y_{t}-\bar{y}\right)^{2} \Rightarrow \sigma_{v}^{2}\left(\eta_{2}-\eta_{1}\right)^{2}
\end{aligned}
$$

and

$$
T^{-2} \sum_{t} x_{t} y_{t} \Rightarrow \sigma_{w} \sigma_{v} \Psi_{11}
$$

where

$$
\Psi_{i j}=\int_{0}^{1}(W(r))^{i}(V(r))^{j} d r=\sigma_{v}^{k} \eta_{k} ; \quad i, j=1,2,3,4, \ldots \ldots
$$

\section{ASYMPTOTIC BEHAVIOUR OF AN OMNIBUS TEST FOR NORMALITY}

Omnibus tests for departures from normality, based on the standardized third and fourth sample moments, have a long tradition dating back at least to Geary (1947a, 1947b). Classic contributions include those of D’Agostino and Pearson (1973) and Bowman and Shenton (1975). Jarque and Bera (1980) proposed a Lagrange multiplier test for normality of the errors in a regression model, and subsequently [Jarque and Bera (1987)] proved that their test is identical to the omnibus test of Bowman and Shenton (1975). In the context of regression residuals, the omnibus test statistic is

$$
O M=(T / 6)\left[m_{3}+\left(m_{4}-3\right)^{2} / 4\right]
$$


where

$$
\begin{aligned}
& m_{3}=\left[T^{-1} \sum_{t}\left(\hat{u}_{t}-\bar{u}\right)^{3} / s^{3}\right]^{2} \\
& m_{4}=T^{-1} \sum_{t}\left(\hat{u}_{t}-\bar{u}\right)^{4} / s^{4}
\end{aligned}
$$

and

$$
s^{2}=T^{-1} \sum_{t}\left(\widehat{u}_{t}-\bar{u}\right)^{2} .
$$

If the model includes an intercept, then of course $\bar{u}=0$, and for a regression model with stationary data, the limiting null distribution of $O M$ is $\chi_{2}^{2}$. However, in the case of a spurious regression the situation is fundamentally different.

\section{Theorem 1}

When applied to the spurious regression model (1), $\left(T^{-1} O M\right)$ converges weakly as, $T \uparrow \infty$ and so $O M$ itself diverges at the rate " $T$ ".

Proof

From Phillips (1986, pp. 330-331):

$$
\widehat{\beta} \Rightarrow\left(\sigma_{v} / \sigma_{w}\right)\left[\left(\Psi_{11}-\xi_{1} \eta_{1}\right) /\left(\xi_{2}-\xi_{1}^{2}\right)\right]=\left(\sigma_{v} / \sigma_{w}\right) \theta \text {, say }
$$

and

$$
T^{-2} s^{2} \Rightarrow \sigma_{v}^{2}\left[\eta_{2}-\eta_{1}^{2}-\theta^{2}\left(\xi_{2}-\xi_{1}^{2}\right)\right] .
$$

So, by the Continuous Mapping Theorem [e.g., Billingsley (1968, pp. 30-31)],

$$
\widehat{\beta}^{k} \Rightarrow\left(\sigma_{v} / \sigma_{w}\right)^{k} \theta^{k} ; \quad k=1,2,3, \ldots \ldots .
$$

and

$$
T^{-k} s^{2 k} \Rightarrow \sigma_{v}^{2 k}\left[\eta_{2}-\eta_{1}^{2}-\theta^{2}\left(\xi_{2}-\xi_{1}^{2}\right)\right]^{k} ; \quad k=1,2,3, \ldots \ldots .
$$

First, consider $m_{3}$ in (13). Defining $y_{t}^{*}=\left(y_{t}-\bar{y}\right)$ and $x_{t}^{*}=\left(x_{t}-\bar{x}\right)$, note that

$$
\sum_{t} \hat{u}_{t}^{3}=\sum_{t} y_{t}^{* 3}-3 \widehat{\beta} \sum_{t} y_{t}^{*_{2}} x_{t}^{*}+3 \widehat{\beta}^{2} \sum_{t} y_{t}^{*} x_{t}^{*_{2}}=\widehat{\beta}^{3} \sum_{t} x_{t}^{*_{3}} .
$$


So, applying the Continuous Mapping Theorem to (20), and using results (18) and (A.1) - (A.4) from the Appendix, we see that the quantity $T^{-5 / 2} \sum_{t} \hat{u}_{t}^{3}$ converges weakly as $T \uparrow \infty$. Finally, using this result and (19) (with $k=3$ ), and applying the Continuous Mapping Theorem to the terms in (13), we see that $m_{3}$ converges weakly with increasing " $T$ ".

Second, consider $m_{4}$ in (14), and note that

$$
\sum_{t} \hat{u}_{t}^{4}=\sum_{t} y_{t}^{* 4}+6 \widehat{\beta}^{2} \sum_{t} y_{t}^{* 2} x_{t}^{* 2}=2 \widehat{\beta} \sum_{t} y_{t}^{* 3} x_{t}^{*}-4 \widehat{\beta}^{3} \sum_{t} y_{t}^{*} x_{t}^{* 4} .
$$

Again, applying the Continuous Mapping Theorem to (21), and using results (18) and (A.5) - (A.9) from the Appendix, the quantity $T^{-3} \sum_{t} \widehat{u}_{t}^{4}$ converges weakly as $T \uparrow \infty$. Finally, using this result and (19) (with $k=4$ ), and applying the Continuous Mapping Theorem to the terms in (13), we see that $m_{4}$ converges weakly with increasing " $T$ ”. Finally, it follows immediately from (12) that $T^{1} O M$ converges weakly, so $O M$ diverges at the rate " $T$ " as $T \uparrow \infty$.

The implication of this result is analogous to that associated with Phillips' (1986, pp. 333-334) result that $(T \times D W)$ converges weakly in the case of a spurious regression, and hence $D W$ itself has a zero probability limit as $T \uparrow \infty$. That is, testing for serial independence or for normality in the errors of a spurious regression will always lead to a rejection of the associated null hypotheses, for large enough $T$, whether these hypotheses are false or true. If a spurious regression is inadvertently estimated, these results may provide an ex post signal to this effect. It should also be noted that the result in Theorem 1 is independent of the initial values and distributions of $v_{t}$ and $w_{t}$ in (2) and (3). In particular, these random errors need not be normally distributed. Table 1 presents some Monte Carlo evidence to illustrate this point, and the results there also demonstrate the rate of divergence of the OM statistic as $T \uparrow \infty$. The Monte Carlo experiment involved 5,000 replications with the values of $v_{t}$ and $w_{t}$ generated as standard normal, uniform $(0,1)$, the inverse of standard normal, and log-normal independent random variables. The simulations were conducted using the SHAZAM (2001) econometrics package. 


\section{ASYMPTOTIC BEHAVIOUR OF THE BREUSCH-PAGAN-GODFREY TEST FOR HOMOSKEDASTICITY}

As is well known, many of the familiar tests for the homoskedasticity of regression errors can be formulated as Lagrange multiplier (LM) tests. For example, see Breusch and Pagan (1980) and Godfrey (1988). One simple example of the Breusch-Pagan-Godfrey (BPG) test involves an alternative hypothesis in which the regression error's variance is proportional to a linear combination of the regressors. For the simple regression model, the implementation of the test involves obtaining the OLS residuals, $\widehat{u}_{t}$, from (1), and then fitting the following auxiliary regression:

$$
\widehat{u}_{t}^{2}=a+b x_{t}+\varepsilon_{t} .
$$

Let $\widehat{a}$ and $\widehat{b}$ be the OLS estimators of $a$ and $b$, and as before let $s^{2}=T^{-1} \sum_{t} \widehat{u}_{t}^{2}$. Then the coefficient of determination associated with the estimation of (31) can be expressed as:

$$
R^{2}=\left[\widehat{b}^{2} \sum_{t} x_{t}^{* 2}\right] /\left[\sum_{t}\left(\hat{u}_{t}-s^{2}\right)^{2}\right]
$$

and an $L M$ test of the homoskedasticity of the errors in (1) can be constructed using ( $\left.T R^{2}\right)$. For this model, if the variables in (1) were stationary then the test statistic would converge in distribution to $\chi_{1}^{2}$ if the null hypothesis were true. As is discussed by Godfrey (1988, Chap. 4) and Greene (2000, pp.509-510), an asymptotically equivalent $L M$ test can be based on the statistic (SSR / 2), where "SSR" denotes the "regression" ("explained") sum of the squares from OLS estimation of the model

$$
\left(\widehat{u}_{t}^{2} / s^{2}\right)=a^{\prime}+b^{\prime} x_{t}+\varepsilon_{t}
$$

In the case of a spurious regression, these two test statistics no longer converge in distribution to $\chi_{1}^{2}$ under the null of homoskedasticity. As was the case for the $O M$ test for normality of the errors, the statistics for both the $\left(T R^{2}\right)$ and (SSR / 2) variants of the $L M$ test diverge in distribution as $T \uparrow \infty$, as we now show. 


\section{Theorem 2}

When applied to the spurious regression model (1), $R^{2}$ defined in (24) converges weakly as $T \uparrow \infty$, and so $T R^{2}$ diverges at the rate " $T$ ”.

Proof

We can write (24) as

$$
R^{2}=\left[\left(T^{-1 / 2} \widehat{b}\right)^{2}\left(T^{-2} \sum_{t} x_{t}^{* 2}\right)\right] /\left[T^{-3} \sum_{t}\left(\widehat{u}_{t}-s^{2}\right)^{2}\right] .
$$

First, note that

$$
\begin{aligned}
T^{-1 / 2} \widehat{b} & =\left[T^{-5 / 2}\left(\sum_{t} x_{t}^{*} \widehat{u}_{t}^{2}-s^{2} \sum_{t} x_{t}^{*}\right)\right] /\left[T^{-2} \sum_{t} x_{t}^{* 2}\right] \\
& =\left[T^{-5 / 2} \sum_{t} x_{t}^{*} \widehat{u}_{t}^{2}\right] /\left[T^{-2} \sum_{t} x_{t}^{* 2}\right]
\end{aligned}
$$

so,

$$
R^{2}=\left[\left(T^{-5 / 2} \sum_{t} x_{t}^{*} \hat{u}_{t}^{2}\right)^{2} /\left(T^{-2} \sum_{t} x_{t}^{* 2}\right)\right] /\left[T^{-3} \sum_{t}\left(\widehat{u}_{t}-s^{2}\right)^{2}\right] .
$$

Now, note that

$$
T^{-3} \sum_{t}\left(\widehat{u}_{t}^{2}-s^{2}\right)^{2}=T^{-3} \sum_{t} \widehat{u}_{t}^{4}+T^{-2} s^{4}-2 T^{-1} s^{2}\left(T^{-2} \sum_{t} \widehat{u}_{t}^{2}\right)
$$

and so using (17) - (19), (21) and results (A.5) - (A.9) from the Appendix, the expression in (28) converges weakly as $T \uparrow \infty$, by the Continuous Mapping Theorem.

Further, we can write

$$
T^{-5 / 2} \sum_{t} x_{t}^{*} \widehat{u}_{t}^{2}=T^{-5 / 2}\left[\sum_{t} x_{t}^{*} y_{t}^{* 2}+\widehat{b}^{2} \sum_{t} x_{t}^{* 3}-2 \bar{b} \sum_{t} x_{t}^{* 2} y_{t}^{*}\right],
$$

and by using (18), (A.1), (A.3) and (A.4), the expression in (27) also converges weakly as $T \uparrow \infty$. Finally, using (8), (28) and (29), the Continuous Mapping Theorem ensures the weak convergence of 
$R^{2}$ in (27). Accordingly, $\left(T R^{2}\right)$ diverges at the rate " $T$ ” as $T \uparrow \infty$.

\section{Theorem 3}

When applied to the spurious regression model (1), the statistic $\left(T^{-1} S S R\right)$ converges weakly as $T \uparrow \infty$, and so the $L M$ test statistic (SSR / 2) diverges at the rate " $T$ ".

Proof

We can write

$$
S S R=\left(\widehat{b}^{\prime}\right)^{2} \sum_{t} x_{t}^{* 2},
$$

where $\bar{b}^{\prime}$ is the OLS estimator of $b^{\prime}$ in (24). Noting that the sample mean of the dependent variable in (24) is unity, we have

$$
\widehat{b}^{\prime}=\left[\sum_{t} x_{t}^{*}\left(\hat{u}_{t}^{2} / s^{2}\right)\right] /\left[\sum_{t} x_{t}^{* 2}\right]
$$

and so

$$
\left(\bar{b}^{\prime}\right)^{2}=T^{-1}\left[T^{-5 / 2} \sum_{t} x_{t}^{*} \widehat{u}_{t}^{2}\right]^{2} /\left[\left(T^{-1} s^{2}\right) \sum_{t} T^{-2} x_{t}^{* 2}\right]^{2}
$$

and

$$
T^{-1} S S R=\left(T^{-2} \sum_{t} x_{t}^{* 2}\right)\left[T^{-5 / 2} \sum_{t} x_{t}^{*} \widehat{u}_{t}^{2}\right]^{2} /\left[\left(T^{-1} S^{2}\right) \sum_{t} T^{-2} x_{t}^{* 2}\right]^{2} .
$$

So, using results (8), (17) and (29), it follows by the Continuous Mapping Theorem that (SSR / 2) itself diverges at the rate " $T$ " as $T \uparrow \infty$.

From Theorems 2 and 3, we see that however it is formulated, the BPG test will increasingly "discover" hetereoskedasticity if we unwittingly apply it in the context of a spurious regression. Recall from section 2 that $v_{t}$ and $w_{t}$ need not be homoskedastic for our various asymptotic results to hold. So, regardless of whether the null hypothesis under test here is true or false, it will be rejected with increasing probability as the sample size grows. This is illustrated in Tables 2 and 3 for the case 
where the null hypothesis is true, and a nominal 10\% significance level (based on the asymptotic $\chi_{1}^{2}$ distribution that would apply for stationary data) is used. The experimental design is the same as in section 3 above. The rates of divergence of the two test statistics, and the commensurate size distortions for the $L M$ tests, can be seen in these tables for various distributions for the errors. Except for the (SSR / 2) version of the test, with $T=10$ and either normal or uniform errors, there is positive size distortion. As the sample size grows, applying the BPG test in the context of a spurious regression leads one to increasingly come to the wrong conclusion that the errors are heteroskedastic. Although this point has been illustrated here with a very simple alternative hypothesis (namely that the variance of the regression errors is proportional to the sole regressor), it is clear that the same basic result also applies to more general variants of the $B P G$ test in which the error variance is proportional to some linear combination of variables under the alternative hypothesis. These results also apply to either the (SSR / 2$)$ or $\left(T R^{2}\right)$ versions of White's (1980) test for homoskedasticity against an arbitrary heteroskedastic alternative, and to other similar tests.

\section{CONCLUSIONS}

Many of the basic pitfalls associated with the use of non-stationary data in regression analysis have been well documented. In particular, Phillips (1986) exposed the underlying reasons for several observed empirical features of "spurious regressions". Among other things, he showed that the standard $t$-test and $F$-test statistics diverge as $T \uparrow \infty$, and the Durbin-Watson statistic converges to zero in probability. Thus, each of the associated null hypotheses will be rejected with increasing probability as the sample size grows, even though in fact they are actually true. This paper follows this theme and extends these results by considering what will be encountered by an applied researcher who (wisely) undertakes some other common types of regression diagnostic testing, but (unwittingly) does so in the context of a spurious regression model.

Clearly, we should test the data for stationarity (and possible cointegration) before estimating a timeseries regression. However, the low power of unit root tests, and their sensitivity to structural breaks in the data, are well known. Accordingly, in practice there is a real risk that a spurious relationship 
involving non-stationary data will be fitted. A high value for the coefficient of determination, in conjunction with a small value for the Durbin-Watson statistic provides a signal that this has occurred. We have shown that additional such signals emerge when, at the same time, standard tests for the normality and homoskedasticity of the model's error term also lead to clear rejections of these hypotheses.

As the sample size grows, these diagnostic tests will increasingly reject the null hypotheses, as a matter of course. To then conclude that the model needs to be reformulated in order to deal with discovered "problems" associated with the error term would be as spurious as the estimation of the original model itself. Although our formal proofs are set in the context of a simple regression model, it is clear from Phillips (1986, pp.319-322) that they extend directly to the multiple regression model.

\section{ACKNOWLEDGMENT}

I am most grateful to Lauren Dong, Mike Veall, and an anonymous referee for their helpful comments on earlier versions of this paper. 
Table 1

Mean Values and Rejection Rates for the $O M$ Test Statistic

(Monte Carlo Simulation, Data Generating Process Given by Equations (1) - (3),

5000 Repetitions, Nominal 10\% Significance Level) ${ }^{1}$

\begin{tabular}{|c|c|c|c|c|c|c|c|c|}
\hline \multirow[b]{2}{*}{$\mathrm{T}$} & \multicolumn{2}{|c|}{$\mathrm{N}(0,1)$} & \multicolumn{2}{|c|}{$\mathrm{U}(0,1)$} & \multicolumn{2}{|c|}{$[1 / \mathrm{N}(0,1)]$} & \multicolumn{2}{|c|}{$\exp [\mathrm{N}(0,1)]$} \\
\hline & $\begin{array}{l}\mathrm{RR} \\
(\%)\end{array}$ & Mean & $\begin{array}{l}\mathrm{RR} \\
(\%)\end{array}$ & Mean & $\begin{array}{l}\mathrm{RR} \\
(\%)\end{array}$ & Mean & $\begin{array}{l}\mathrm{RR} \\
(\%)\end{array}$ & Mean \\
\hline 10 & 1.34 & 0.91 & 0.54 & 0.78 & 10.02 & 2.03 & 4.24 & 1.28 \\
\hline 20 & 3.36 & 1.45 & 1.34 & 1.08 & 23.06 & 8.22 & 11.26 & 2.90 \\
\hline 50 & 13.84 & 2.97 & 8.06 & 2.30 & 55.64 & 49.38 & 26.94 & 7.09 \\
\hline 100 & 45.78 & 5.95 & 35.10 & 4.48 & 81.08 & 158.42 & 55.28 & 11.37 \\
\hline 250 & 79.68 & 14.29 & 67.84 & 8.77 & 93.54 & 1036.56 & 77.72 & 20.90 \\
\hline 500 & 91.14 & 29.57 & 89.36 & 22.25 & 97.20 & 6594.69 & 91.28 & 31.61 \\
\hline 1000 & 95.90 & 57.94 & 95.34 & 45.02 & 98.74 & 19997.91 & 95.86 & 53.83 \\
\hline 2000 & 98.08 & 121.83 & 97.96 & 90.52 & 99.24 & 92016.16 & 97.74 & 100.63 \\
\hline 5000 & 99.30 & 290.83 & 99.18 & 231.64 & 99.76 & 39813.92 & 99.04 & 233.01 \\
\hline 10000 & 99.50 & 600.22 & 99.50 & 451.83 & 99.82 & 60762.71 & 99.64 & 479.90 \\
\hline
\end{tabular}

1. The $10 \%$ critical value for the Chi Square distribution with 2 degrees of freedom is 4.60517. 'RR' denotes the 'rejection rate' - i.e., the percentage of the 5,000 simulated values for JB that exceeded this critical value. 
Table 2

Mean Values and Rejection Rates for BPG Test Statistic: $T R^{2}$ Version (Monte Carlo Simulation, Data Generating Process Given by Equations (1) - (3), 5000 Repetitions, Nominal 10\% Significance Level) ${ }^{1}$

\begin{tabular}{|c|c|c|c|c|c|c|c|c|}
\hline \multirow[b]{2}{*}{$\mathrm{T}$} & \multicolumn{2}{|c|}{$\mathrm{N}(0,1)$} & \multicolumn{2}{|c|}{$\mathrm{U}(0,1)$} & \multicolumn{2}{|c|}{$[1 / \mathrm{N}(0,1)]$} & \multicolumn{2}{|c|}{$\exp [N(0,1)]$} \\
\hline & $\begin{array}{l}\mathrm{RR} \\
(\%)\end{array}$ & Mean & $\begin{array}{l}\mathrm{RR} \\
(\%)\end{array}$ & Mean & $\begin{array}{l}\text { RR } \\
(\%)\end{array}$ & Mean & $\begin{array}{l}\text { RR } \\
\text { (\%) }\end{array}$ & Mean \\
\hline 10 & 12.68 & 1.14 & 14.30 & 1.20 & 25.32 & 1.86 & 25.36 & 1.74 \\
\hline 20 & 19.54 & 1.55 & 21.58 & 1.59 & 39.58 & 3.17 & 35.54 & 2.60 \\
\hline 50 & 38.88 & 3.24 & 42.86 & 3.56 & 58.76 & 7.52 & 52.28 & 5.30 \\
\hline 100 & 53.14 & 6.26 & 57.94 & 7.00 & 70.80 & 14.98 & 63.82 & 9.49 \\
\hline 250 & 69.82 & 15.44 & 72.94 & 17.57 & 80.80 & 70.70 & 74.96 & 20.76 \\
\hline 500 & 78.00 & 31.54 & 79.94 & 34.37 & 87.54 & 75.01 & 81.06 & 38.27 \\
\hline 1000 & 84.84 & 64.62 & 85.34 & 70.01 & 90.80 & 149.41 & 86.84 & 75.54 \\
\hline 2000 & 88.54 & 123.67 & 90.14 & 140.97 & 93.22 & 298.48 & 91.08 & 147.95 \\
\hline 5000 & 93.34 & 304.76 & 93.06 & 339.70 & 95.80 & 736.72 & 93.50 & 358.96 \\
\hline 10000 & 94.78 & 614.62 & 95.86 & 698.52 & 97.44 & 1575.00 & 94.94 & 675.14 \\
\hline
\end{tabular}

1. The $10 \%$ critical value for the Chi Square distribution with 1degree of freedom is 2.70554. 'RR' denotes the 'rejection rate' - i.e., the percentage of the 5,000 simulated values for BPG that exceeded this critical value. 
Table 3

Mean Values and Rejection Rates for BPG Test Statistic: SSR Version (Monte Carlo Simulation, Data Generating Process Given by Equations (1) - (3), 5000 Repetitions, Nominal 10\% Significance Level) ${ }^{1}$

\begin{tabular}{|c|c|c|c|c|c|c|c|c|}
\hline \multirow[b]{2}{*}{$\mathrm{T}$} & \multicolumn{2}{|c|}{$\mathrm{N}(0,1)$} & \multicolumn{2}{|c|}{$\mathrm{U}(0,1)$} & \multicolumn{2}{|c|}{$[1 / \mathrm{N}(0,1)]$} & \multicolumn{2}{|c|}{$\exp [\mathrm{N}(0,1)]$} \\
\hline & $\begin{array}{l}\mathrm{RR} \\
(\%)\end{array}$ & Mean & $\begin{array}{l}\mathrm{RR} \\
(\%)\end{array}$ & Mean & $\begin{array}{l}\text { RR } \\
\text { (\%) }\end{array}$ & Mean & $\begin{array}{l}\mathrm{RR} \\
(\%)\end{array}$ & Mean \\
\hline 10 & 4.88 & 0.73 & 6.14 & 0.79 & 15.50 & 1.40 & 15.08 & 1.27 \\
\hline 20 & 12.16 & 1.13 & 14.40 & 1.22 & 34.00 & 2.91 & 27.84 & 2.23 \\
\hline 50 & 30.32 & 2.38 & 34.88 & 2.87 & 56.28 & 7.53 & 45.56 & 4.74 \\
\hline 100 & 45.74 & 4.73 & 50.34 & 5.71 & 68.24 & 14.61 & 57.68 & 8.35 \\
\hline 250 & 64.92 & 11.74 & 67.60 & 14.38 & 79.78 & 39.61 & 70.60 & 17.96 \\
\hline 500 & 74.72 & 23.84 & 75.94 & 28.30 & 86.68 & 78.11 & 77.34 & 32.34 \\
\hline 1000 & 82.76 & 48.17 & 82.90 & 56.61 & 90.84 & 153.11 & 84.42 & 63.12 \\
\hline 2000 & 86.46 & 94.37 & 88.16 & 114.92 & 92.80 & 303.22 & 89.56 & 122.28 \\
\hline 5000 & 92.12 & 228.55 & 91.56 & 284.06 & 95.36 & 774.02 & 92.50 & 296.50 \\
\hline 10000 & 93.98 & 460.56 & 94.78 & 570.44 & 97.10 & 1605.40 & 94.02 & 556.36 \\
\hline
\end{tabular}

1. The $10 \%$ critical value for the Chi Square distribution with 1 degree of freedom is 2.70554. 'RR' denotes the 'rejection rate' - i.e., the percentage of the 5,000 simulated values for BPG that exceeded this critical value. 


\section{APPENDIX}

\section{RESULTS USED FOR THE PROOF OF THEOREM 1}

$$
\begin{aligned}
& T^{-5 / 2} \sum_{t} x_{t}^{* 3}=T^{-5 / 2} \sum_{t}\left(x_{t}^{3}-3 x_{t}^{2} \bar{x}+3 x_{t} \bar{x}^{2}-\bar{x}^{3}\right) \\
& =\left(T^{-5 / 2} \sum_{t} x_{t}^{3}\right)-3 T^{-5 / 2}\left(T^{-1} \sum_{t} x_{t}\right)\left(\sum_{t} x_{t}^{2}\right)+3\left(T^{-1} \sum_{t} x_{t}\right)^{2}\left(T^{-5 / 2} \sum_{t} x_{t}\right)-T^{-3 / 2}\left(T^{-1} \sum_{t} x_{t}\right)^{3} \\
& =\left(T^{-5 / 2} \sum_{t} x_{t}^{3}\right)-3 T^{-3 / 2}\left(\sum_{t} x_{t}\right)\left(T^{-2} \sum_{t} x_{t}^{2}\right)+3\left(T^{-3 / 2} \sum_{t} x_{t}\right)^{2}\left(T^{-3 / 2} \sum_{t} x_{t}\right)-\left(T^{-3 / 2} \sum_{t} x_{t}\right)^{3}
\end{aligned}
$$

Then, repeatedly applying result (6) in section 2 (with $k=1,2,3$ ), and appealing to the Continuous Mapping Theorem, we obtain:

$$
T^{-5 / 2} \sum x_{t}^{* 3} \Rightarrow \sigma_{w}^{3}\left[\xi_{3}-3 \xi_{1} \xi_{2}+2 \xi_{1}^{3}\right]
$$

Similarly, the repeated application of result (7) in section 2 yields:

$$
T^{-5 / 2} \sum y_{t}^{* 3} \Rightarrow \sigma_{v}^{3}\left[\eta_{3}-3 \eta_{1} \eta_{2}+2 \eta_{1}^{3}\right]
$$

Also,

$$
\begin{aligned}
T^{-5 / 2} \sum_{t} y_{t}^{* 2} x_{t}^{*}= & T^{-5 / 2}\left(\sum_{t} x_{t} y_{t}^{2}-2 \bar{y} \sum_{t} x_{t} y_{t}+\bar{y}^{2} \sum_{t} x_{t}-\bar{x} \sum_{t} y_{t}^{2}+2 \bar{x} \bar{y} \sum_{t} y_{t}-\sum_{t} \overline{x y}^{2}\right) \\
= & \left(T^{-5 / 2} \sum_{t} x_{t} y_{t}^{2}\right)-2\left(T^{-3 / 2} \sum_{t} y_{t}\right)\left(T^{-2} \sum_{t} x_{t} y_{t}\right)-\left(T^{-3 / 2} \sum_{t} x_{t}\right)\left(T^{-2} \sum_{t} y_{t}^{2}\right) \\
& +2\left(T^{-3 / 2} \sum_{t} x_{t}\right)\left(T^{-3 / 2} \sum_{t} y_{t}\right)^{2}
\end{aligned}
$$

Then, by the Continuous Mapping Theorem, using definition (11) and results (4), (5), (10) in section 2, and generalizing the last of these three results in a natural manner, we get:

$$
T^{-5 / 2} \sum_{t} y_{t}^{* 2} x_{t}^{*} \Rightarrow\left(\sigma_{v}^{2} \sigma_{w}\right)\left[\Psi_{12}-2 \eta_{1} \Psi_{11}-\xi_{1}\left(\eta_{2}+2 \eta_{1}^{2}\right)\right]
$$

Interchanging the $y_{t}^{*}$ and $x_{t}^{*}$ variables in the derivation of (A.3) above, we get:

$$
T^{-5 / 2} \sum_{t} x_{t}^{* 2} y_{t}^{*} \Rightarrow\left(\sigma_{w}^{2} \sigma_{v}\right)\left[\Psi_{21}-2 \xi_{1} \Psi_{11}-\eta_{1}\left(\xi_{2}+2 \xi_{1}^{2}\right)\right]
$$


Next,

$$
\begin{aligned}
T^{-3} \sum_{t} y_{t}^{* 4}= & T^{-3}\left(\sum_{t} y_{t}^{4}-4 \bar{y} \sum_{t} y_{t}^{3}+6 \bar{y}^{2} \sum_{t} y_{t}^{2}-4 \bar{y}^{3} \sum_{t} y_{t}+\sum_{t} \bar{y}^{4}\right) \\
= & \left(T^{-3} \sum_{t} y_{t}^{4}\right)-4\left(T^{-3 / 2} \sum_{t} y_{t}\right)\left(T^{-5 / 2} \sum_{t} y_{t}^{3}\right)+6\left(T^{-5 / 2} \sum_{t} y_{t}\right)^{2}\left(T^{-2} \sum_{t} y_{t}^{2}\right) \\
& -4\left(T^{-3 / 2} \sum_{t} y_{t}\right)^{4}+\left(T^{-3 / 2} \sum_{t} y_{t}\right)^{4} .
\end{aligned}
$$

Then, using the Continuous Mapping Theorem, result (7) from section 2 repeatedly, and gathering terms, we get:

$$
T^{-3} \sum_{t} y_{t}^{* 4} \Rightarrow \sigma_{v}^{4}\left[\eta_{4}-4 \eta_{1} \eta_{3}+6 \eta_{1}^{2} \eta_{2}-3 \eta_{1}^{4}\right]
$$

Interchanging the $y_{t}^{*}$ and $x_{t}^{*}$ variables in the derivation of (A.5) above, we get:

$$
T^{-3} \sum_{t} x_{t}^{* 4} \Rightarrow \sigma_{w}^{4}\left[\xi_{4}-4 \xi_{1} \xi_{3}+6 \xi_{1}^{2} \xi_{2}-3 \xi_{1}^{4}\right]
$$

Next,

$$
\begin{aligned}
& T^{-3} \sum_{t} y_{t}^{* 2} x_{t}^{* 2}=T^{-3 / 2} \sum_{t}\left(y_{t}^{2} x_{t}^{2}-2 \bar{x} x_{t} y_{t}+\bar{x}^{2} y_{t}^{2}-2 \bar{y} y_{t} x_{t}^{2}+4 \overline{x y} x_{t} y_{t}-2 \overline{y x}^{2} y_{t}\right. \\
& \left.+\bar{y}^{2} x_{t}^{2}-2 \overline{x y}^{2} x_{t}+\bar{x}^{2} \bar{y}^{2}\right) \\
& =\left(T^{-3} \sum_{t} x_{t}^{2} y_{t}^{2}\right)-2\left(T^{-3 / 2} \sum_{t} x_{t}\right)\left(T^{-5 / 2} \sum_{t} x_{t} y_{t}^{2}\right)+\left(T^{-3 / 2} \sum_{t} x_{t}\right)^{2}\left(T^{-2} \sum_{t} y_{t}^{2}\right) \\
& -2\left(T^{-3 / 2} \sum_{t} y_{t}\right)\left(T^{-5 / 2} \sum_{t} y x_{t}^{2}\right)+4\left(T^{-3 / 2} \sum_{t} x_{t}\right)\left(T^{-3 / 2} \sum_{t} y_{t}\right)\left(T^{-2} \sum_{t} x_{t} y_{t}\right) \\
& -2\left(T^{-3 / 2} \sum_{t} y_{t}\right)^{2}\left(T^{-3 / 2} \sum_{t} x_{t}\right)^{2}+\left(T^{-3 / 2} \sum_{t} y_{t}\right)^{2}\left(T^{-2} \sum_{t} x_{t}^{2}\right) \\
& -2\left(T^{-3 / 2} \sum_{t} x_{t}\right)^{2}\left(T^{-3 / 2} \sum_{t} y_{t}\right)^{2}+\left(T^{-3 / 2} \sum_{t} x_{t}\right)^{2}\left(T^{-3 / 2} \sum_{t} y_{t}\right)^{2} .
\end{aligned}
$$

By the Continuous Mapping Theorem, using definition (11) and results (6), (7) and (10) in section 2, and generalizing the last of these three results in a natural manner, we get: 


$$
T^{-3} \sum_{t} y_{t}^{* 2} x_{t}^{* 2} \Rightarrow\left(\sigma_{v}^{2} \sigma_{w}^{2}\right)\left[\Psi_{22}-2 \xi_{1} \Psi_{12}+\xi_{1}^{2} \eta_{2}+\eta_{1}^{2} \xi_{2}-2 \eta_{1} \Psi_{21}+4 \xi_{1} \eta_{1} \Psi_{11}-3 \eta_{1}^{2} \xi_{1}^{2}\right]
$$

Similarly,

$$
\begin{aligned}
T^{=3} \sum_{t} y_{t}^{* 3} x_{t}^{*} & =T^{-3}\left(\sum_{t} x_{t} y_{t}^{3}-\bar{y} \sum_{t} x_{t} y_{t}^{2}+\bar{x} \sum_{t} y_{t}^{3}+\overline{x y} \sum_{t} y_{t}^{2}-2 \bar{y} \sum_{t} x_{t} y_{t}^{2}+2 \bar{y} \sum_{t} x_{t} y_{t}^{2}\right. \\
& +2 \bar{y}^{2} \sum_{t} x_{t} y_{t}+2 \overline{x y} \sum_{t} y_{t}^{2}-2 \overline{x y}^{2} \sum_{t} y_{t}+\bar{y}^{2} \sum_{t} x_{t} y_{t}-\bar{y}^{2} \bar{x} \sum_{t} y_{t}+T x y \\
& =\left(T^{-3} \sum_{t} x_{t} y_{t}^{3}\right)-3\left(T^{-3 / 2} \sum_{t} y_{t}\right)\left(T^{-5 / 2} \sum_{t} x_{t} y_{t}^{2}\right)-\left(T^{-3 / 2} \sum_{t} x_{t}\right)\left(T^{-5 / 2} \sum_{t} y_{t}^{3}\right) \\
& +3\left(T^{-3 / 2} \sum_{t} x_{t}\right)\left(T^{-3 / 2} \sum_{t} y_{t}\right)\left(T^{-2} \sum_{t} y_{t}^{2}\right)+3\left(T^{-3 / 2} \sum_{t} y_{t}\right)^{2}\left(T^{-2} \sum_{t} x_{t} y_{t}\right) \\
& -3\left(T^{-3 / 1} \sum_{t} x_{t}\right)\left(T^{-3 / 2} \sum_{t} y_{t}\right)^{3} .
\end{aligned}
$$

Again, by the Continuous Mapping Theorem, using definition (11) and results (7) and (10) in section 2, and generalizing the last of these three results in a natural manner, we get:

$$
T^{-3} \sum_{t} y_{t}^{* 3} x_{t}^{*} \Rightarrow\left(\sigma_{v}^{3} \sigma_{w}\right)\left[\Psi_{13}-3 \eta_{1} \Psi_{12}-\xi_{1} \eta_{3}+3 \eta_{1}^{2} \Psi_{11}-3 \xi_{1} \eta_{1}^{3}+3 \xi_{1} \eta_{1} \eta_{2}\right]
$$

Finally, interchanging the $y_{t}^{*}$ and $x_{t}^{*}$ variables in the derivation of (A.8) above, and using result (6), we get:

$$
T^{-3} \sum_{t} x_{t}^{* 3} y_{t}^{*} \Rightarrow\left(\sigma_{v} \sigma_{w}^{3}\right)\left[\Psi_{31}-3 \xi_{1} \Psi_{21}-\eta_{1} \xi_{3}+3 \xi_{1}^{2} \Psi_{11}-3 \eta_{1} \xi_{1}^{3}+3 \eta_{1} \xi_{1} \xi_{2}\right]
$$




\section{REFERENCES}

Billingsley, D. P. (1968). Convergence of Probability Measures. New York: Wiley.

Bowman, K. O., Shenton, L. R. (1975). Omnibus test contours for departures from normality based on $\sqrt{b_{1}}$ and $\mathrm{b}_{2}$. Biometrika 62: 243-250.

Breusch, T. S., Pagan, A. R. (1980). The Lagrange multiplier test and its applications to model specification in econometrics. Review of Economic Studies 47: 239-253.

D’Agostino, R., Pearson, E. S. (1973). Tests for departure from normality. Empirical results for the distributions of $b_{2}$ and $\sqrt{b_{1}}$. Biometrika 60: 613-622.

Geary, R. C. (1947a). Frequency distribution of $\sqrt{b_{1}}$. Biometrika 34: 68-97.

Geary, R. C. (1947b). Testing for normality. Biometrika 34: 209-242.

Godfrey, L. G. (1988). Misspecification Tests in Econometrics: The Lagrange Multiplier Principle and other Methods. Cambridge: Cambridge University Press.

Granger, C. W. J., Newbold, P. (1974). Spurious regressions in econometrics. Journal of Econometrics 2: 111120.

Granger, C. W. J., Newbold, P. (1977). Forecasting Economic Time Series. New York: Academic Press. Greene, W. H. (2000). Econometric Analysis, $4^{\text {th }}$. ed. Upper Saddle River NJ: Academic Press.

Hamilton, J. B. (1994. Time Series Analysis. Princeton NJ; Princeton University Press.

Jarque, C., Bera, A. (1980). Efficient tests for normality, homoskedasticity and serial independence of regression residuals. Economics Letters 6: 255-259.

Jarque, C., Bera, A. (1987). A test for normality of observations and regression residuals. International Statistical Review 55: 163-172.

McLeish, D. L. (1975). A Maximal inequality and dependent strong laws. Annals of Probability 3: 829-839.

Phillips, P. C. B. (1986). Understanding spurious regressions in econometrics, Journal of Econometrics 33: 311-340.

Plosser, C. I. and G. W. Schwert (1978), Money, income and sunspots: Measuring economic relationships and the effects of differencing, Journal of Monetary Economics, 4, 637-660.

SHAZAM (2001). SHAZAM econometrics Package: Users' Reference Manual, Version 9. New York: McGraw-Hill.

White, H. (1980). A heteroskedasticity-consistent covariance matrix estimator and a direct test for heteroskedasticity. Econometrica 48: 817-838. 